\section{Prognostic prediction by liver tissue proteomic profiling in patients with colorectal liver metastases; rule of thumb}

\author{
Response to: Reyes A, Marti J, Marfà S et al. Prognostic prediction by liver tissue \\ proteomic profiling in patients with colorectal liver metastases. Future Oncol. 13(10), \\ 875-882 (2017)
}

\section{Siamak Sabour*}

First draft submitted: 21 January 2017; Accepted for publication: 9 March 2017; Published online: 23 June 2017

I was interested to read the paper by Reyes and colleagues published in Future Oncology January 2017. The purpose of the paper was to obtain proteomic profiles in patients with colorectal liver metastases (CRLM) and identify the relationship between profiles and the prognosis of CRLM patients [1]. They performed prognosis prediction (favorable or unfavorable according to Fong's score) by a classification and regression tree algorithms of surface-enhanced laser desorption/ionization TOF-MS proteomic profiles from cryopreserved CRLM (patients) and normal liver tissue (controls). Based on their results, the protein peak $7371 \mathrm{~m} / \mathrm{z}$ showed the clearest differences between CRLM and control groups $(94.1 \%$ sensitivity, $100 \%$ specificity and $\mathrm{p}<0.001)$.

As a rule of thumb, for prediction studies, we need two different cohort datasets. Otherwise, at least one cohort dataset is required splitting that to develop our prediction model and then to validate it. Without validation of prediction, most of the times, misleading results will be the main outcome of such research [2-6].

Moreover, statistically significant does not necessarily mean clinically important results for clinical decision making. In clinical researches, the strength of the relation is more important than p-value [2-6]. Finally, in prediction studies, we must assess interaction between variables. It is good to know that qualitative interaction can extremely change the result of prediction models [2-6].

The authors conclude that proteomic profiling in liver samples using classification and regression tree algorithms is a promising technique to differentiate healthy subjects from CRLM patients and to classify the severity of CRLM patients. Such conclusion should be supported by the abovementioned rules in prediction studies.

\section{Disclaimer}

The opinions expressed in this interview are those of the interviewees and do not necessarily reflect the views of Future Medicine Ltd.

\section{Financial \& competing interests disclosure}

The author has no relevant affiliations or financial involvement with any organization or entity with a financial interest in or financial conflict with the subject matter or materials discussed in the manuscript. This includes employment, consultancies, honoraria, stock ownership or options, expert testimony, grants or patents received or pending, or royalties.

No writing assistance was utilized in the production of this manuscript.

\section{KEYWORDS}

- biomarker • colorectal cancer • liver metastases

- outcomes research

- prognosis $\bullet$ proteomic analysis 


\section{LETTER TO THE EDITOR Sabour}

\section{References}

1 Reyes A, Marti J, Marfà S et al. Prognostic prediction by liver tissue proteomic profiling in patients with colorectal liver metastases. Future Oncol. 13(10), 875-882 (2017)

2 Rothman KJ, Sander Greenland, Timothy L. Lash. Cohort studies. In: Rothman KJ.

Modern Epidemiology (3rd Edition). Kenneth
J. Rothmanm, Sander Greenland (Eds). Lippincott Williams \& Wilkins, MD, USA (2008).

3 Sabour S. Prediction of preterm delivery using levels of VEGF and leptin in amniotic fluid from the second trimester: prediction rules. Arch. Gynecol. Obstet. 291(4), 719 (2015).

4 Sabour S. Prediction of 3-dimensional pharyngeal airway changes after orthognathic surgery: a methodological issue. Am. J. Orthod. Dentofacial Orthop. 147(1), 8 (2015).

5 Sabour S. Obesity predictors in people with chronic spinal cord injury: a common mistake. J. Res. Med. Sci. 19(1), 80 (2014).

6 Sabour S, Ghassemi F. Predictive value of confocal scanning laser for the onset of visual field loss. Ophthalmology 120(6), e31-e32 (2013). 